\title{
Singular degenerate problems occurring in biosorption process
}

\author{
Aida Sahmurova' and Veli B Shakhmurov ${ }^{2 *}$
}

\section{"Correspondence:}

veli.sahmurov@okan.edu.tr

${ }^{2}$ Department of Mechanical Engineering, Okan University,

Akfirat, Tuzla, Istanbul 34959, Turkey Full list of author information is available at the end of the article

\begin{abstract}
The boundary value problems for singular degenerate arbitrary order differential-operator equations with variable coefficients are considered. The uniform coercivity properties of ordinary and partial differential equations with small parameters are derived in abstract $L_{p}$ spaces. It is shown that corresponding differential operators are positive and also are generators of analytic semigroups. In application, well-posedeness of the Cauchy problem for an abstract parabolic equation and systems of parabolic equations are studied in mixed $L_{\mathbf{p}}$ spaces. These problems occur in fluid mechanics and environmental engineering.

MSC: $34 \mathrm{G} 10 ; 35 J 25 ; 35 J 70$
\end{abstract}

Keywords: differential-operator equations; degenerate equations; semigroups of operators; Banach-valued function spaces; coercive problems; operator-valued Fourier multipliers; interpolation of Banach spaces

\section{Introduction}

Boundary value problems (BVPs) for differential-operator equations (DOEs) in $H$-valued (Hilbert space valued) function spaces have been studied extensively by many researchers (see, e.g., [1-14] and the references therein). A comprehensive introduction to DOEs and historical references may be found in [6] and [14]. The maximal regularity properties for DOEs have been studied, e.g., in [3,10-19].

In this work, singular degenerate BVPs for arbitrary order DOEs with parameters are considered. This problem has numerous applications. The parameter-dependent BVPs occur in different situations of fluid mechanics and environmental engineering etc.

In Section 2, the BVP for the following singular degenerate ordinary DOE with a small parameter:

$$
L u=(-1)^{m} t a(x) u^{[2 m]}+A(x) u+\sum_{k=0}^{2 m-1} t^{\frac{k}{2 m}} A_{k}(x) u^{[k]}=f(x),
$$

is considered, where

$$
D_{x}^{[i]} u=u^{[i]}(x)=\left[x^{\gamma} \frac{d}{d x}\right]^{i} u(x), \quad x \in(0,1)
$$

$t$ is a small parameter, $a(x)$ is a complex-valued function, $\gamma>1, A=A(x)$ is a principal, $A_{1}=A_{1}(x)$ and $A_{2}=A_{2}(x)$ are subordinate linear operators in a Banach space $E$.

\section{Springer}

(c) 2013 Sahmurova and Shakhmurov; licensee Springer. This is an Open Access article distributed under the terms of the Creative Commons Attribution License (http://creativecommons.org/licenses/by/2.0), which permits unrestricted use, distribution, and reproduction in any medium, provided the original work is properly cited. 
Several conditions for the uniform coercivity and the resolvent estimates for this problem are given in abstract $L_{p}$-spaces. We prove that the problem has a unique solution $u \in W_{p, \gamma}^{[2]}(0,1 ; E(A), E)$ for $f \in L_{p}(0,1 ; E),|\arg \lambda| \leq \varphi, 0 \leq \varphi<\pi$ with sufficiently large $|\lambda|$ and the following uniform coercive estimate holds:

$$
\sum_{i=0}^{2}|\lambda|^{1-\frac{i}{2}} t^{\sigma_{i}}\left\|u^{[i]}\right\|_{L_{p}(0,1 ; E)}+\|A u\|_{L_{p}(0,1 ; E)} \leq C\|f\|_{L_{p}(0,1 ; E)},
$$

where

$$
\sigma_{i}=\frac{1}{2 p(\gamma-1)}+\frac{i}{2}
$$

In Section 3, the partial DOE with small parameters

$$
\begin{aligned}
& \sum_{k=1}^{n}(-1)^{m_{k}} t_{k} a_{k}(x) D_{x_{k}}^{\left[2 m_{k}\right]} u+A(x) u+\sum_{|\alpha: 2 m|<1} \prod_{k=1}^{n} t_{k}^{\frac{\alpha_{k}}{2 m_{k}}} A_{\alpha}(x) D^{[\alpha]} u=f(x), \\
& \quad x=\left(x_{1}, x_{2}, \ldots, x_{n}\right) \in G
\end{aligned}
$$

is considered in a mixed $L_{\mathbf{p}}(G ; E)$ space, where $a_{k}$ are complex-valued functions, $A$ and $A_{\alpha}$ are linear operators in $E, \lambda$ is a complex and $t_{k}$ are positive parameters, $G$ is an $n$ dimensional rectangular domain, $\mathbf{p}=\left(p_{1}, p_{2}, \ldots, p_{n}\right)$. Here we prove that for $f \in L_{\mathbf{p}}(G ; E)$, $|\arg \lambda| \leq \varphi$ with sufficiently large $|\lambda|$, this problem has a unique solution $u$ that belongs to the Sobolev space $W_{\mathbf{p}}^{[2]}(G ; E(A), E)$ with a mixed $\mathbf{p}$ norm and the following coercive uniform estimate holds:

$$
\sum_{k=1}^{n} \sum_{i=0}^{2 m_{k}}|\lambda|^{1-\frac{i}{2 m_{k}}} t_{k}^{\sigma_{k i}}\left\|D_{x_{k}}^{[i]} u\right\|_{L_{\mathbf{p}}(G ; E)}+\|A u\|_{L_{\mathbf{p}}(G ; E)} \leq C\|f\|_{L_{\mathbf{p}}(G ; E)},
$$

where

$$
D_{x_{k}}^{[i]}=\left[x_{k}^{\alpha_{k}} \frac{\partial}{\partial x_{k}}\right]^{i}, \quad \alpha_{k}>1, \quad \sigma_{k i}=\frac{1}{2 m_{k} p\left(\alpha_{k}-1\right)}+\frac{i}{2 m_{k}}, \quad k=1,2, \ldots, n .
$$

In Section 4, the uniform well-posedeness of the mixed problem for the following singular degenerate abstract parabolic equation:

$$
\frac{\partial u}{\partial y}+\sum_{k=1}^{n}(-1)^{m_{k}} t_{k} a_{k}(x) D_{x_{k}}^{\left[2 m_{k}\right]} u+A(x) u=f(x, y)
$$

is obtained. Particularly, the above problem occurs in atmospheric dispersion of pollutants and evolution models for phytoremediation of metals from soils. In application, particularly, by taking $E=R^{3}, A(x)=\left[a_{i j}(x)\right], u=\left(u_{1}, u_{2}, u_{3}\right), i, j=1,2,3$, we consider the mixed problem for the system of the following parabolic equations with parameters:

$$
\frac{\partial u_{i}}{\partial y}+\sum_{k=1}^{n}(-1)^{m_{k}} t_{k} a_{k}(x) D_{x_{k}}^{\left[2 m_{k}\right]} u_{i}+\sum_{j=1}^{3} a_{i j}(x) u_{j}=f_{i}(x, y)
$$


which arises in phytoremediation process, where $a_{i j}(x)$ are real-valued functions and $f_{i}(x, y)$ are data. The maximal regularity properties of this problem are studied. Note that the maximal regularity properties for undegenerate DOEs were investigated, e.g., in [110, 14-16, 19, 20]. Regular degenerate DOEs in Banach spaces were treated in [11-13, 15, 17-19, 21]. It should be noted that contrary to these results, here high-order singular degenerated BVPs with small parameters are considered. Moreover, principal coefficients depend on space variables. The proofs are based on abstract harmonic analysis, operator theory, interpolation of Banach spaces, theory of semigroups of linear operators, microlocal analysis, embedding and trace theorems in vector-valued Sobolev-Lions spaces.

\section{Notations and background}

Let $\gamma=\gamma(x), x=\left(x_{1}, x_{2}, \ldots, x_{n}\right)$ be a positive measurable function on a domain $\Omega \subset R^{n}$. $L_{p, \gamma}(\Omega ; E)$ denotes the space of strongly measurable $E$-valued functions that are defined on $\Omega$ with the norm

$$
\|f\|_{L_{p, \gamma}}=\|f\|_{L_{p, \gamma}(\Omega ; E)}=\left(\int\|f(x)\|_{E}^{p} \gamma(x) d x\right)^{\frac{1}{p}}, \quad 1 \leq p<\infty .
$$

For $\gamma(x) \equiv 1$, the space $L_{p, \gamma}(\Omega ; E)$ will be denoted by $L_{p}=L_{p}(\Omega ; E)$.

The Banach space $E$ is called a UMD-space if the Hilbert operator

$$
(H f)(x)=\lim _{\varepsilon \rightarrow 0} \int_{|x-y|>\varepsilon} \frac{f(y)}{x-y} d y
$$

is bounded in $L_{p}(R, E), p \in(1, \infty)$ (see, e.g., [22]). UMD spaces include, e.g., $L_{p}, l_{p}$ spaces and Lorentz spaces $L_{p q}, p, q \in(1, \infty)$.

Let $C$ denote the set of complex numbers and

$$
S_{\varphi}=\{\lambda ; \lambda \in C,|\arg \lambda| \leq \varphi\} \cup\{0\}, \quad 0 \leq \varphi<\pi .
$$

A linear operator $A$ is said to be $\varphi$-positive in a Banach space $E$ with bound $M>0$ if $D(A)$ is dense on $E$ and $\left\|(A+\lambda I)^{-1}\right\|_{B(E)} \leq M(1+|\lambda|)^{-1}$ for any $\lambda \in S_{\varphi}, 0 \leq \varphi<\pi$, where $I$ is the identity operator in $E, B(E)$ is the space of bounded linear operators in $E$. Sometimes $A+\lambda I$ will be written as $A+\lambda$ and denoted by $A_{\lambda}$. It is known [23, Section 1.15.1] that a positive operator $A$ has well-defined fractional powers $A^{\theta}$. Let $E\left(A^{\theta}\right)$ denote the space $D\left(A^{\theta}\right)$ with the norm

$$
\|u\|_{E\left(A^{\theta}\right)}=\left(\|u\|^{p}+\left\|A^{\theta} u\right\|^{p}\right)^{\frac{1}{p}}, \quad 1 \leq p<\infty, 0<\theta<\infty .
$$

Let $E_{1}$ and $E_{2}$ be two Banach spaces continuously embedded in a locally convex space. By $\left(E_{1}, E_{2}\right)_{\theta, p}, 0<\theta<1,1 \leq p \leq \infty$, we denote the interpolation spaces obtained from $\left\{E_{1}, E_{2}\right\}$ by the $K$-method [23, Section 1.3.2].

Let $C(\Omega ; E)$ denote the space of $E$-valued uniformly bounded continuous functions on the domain $\Omega \subset R^{n}$.

Let $N$ denote the set of natural numbers and $\left\{r_{j}\right\}$ be a sequence of independent symmetric $\{-1,1\}$-valued random variables on $[0,1]$ (see [22]). A set $W_{h} \subset B\left(E_{1}, E_{2}\right)$ is called 
uniform $R$-bounded with respect to $h$ (see, e.g., [16]) if there is a constant $C$ independent of $h \in Q \subset R$ such that for all $T_{1}(h), T_{2}(h), \ldots, T_{m}(h) \in W_{h}$ and $u_{1}, u_{2}, \ldots, u_{m} \in E_{1}, m \in N$,

$$
\int_{0}^{1}\left\|\sum_{j=1}^{m} r_{j}(y) T_{j}(h) u_{j}\right\|_{E_{2}} d y \leq C \int_{0}^{1}\left\|\sum_{j=1}^{m} r_{j}(y) u_{j}\right\|_{E_{1}} d y .
$$

The smallest $C$ for which the above estimate holds is called an $R$-bound of the collection $W_{h}$ and is denoted by $R\left(W_{h}\right)$.

A $\varphi$-positive operator $A$ is said to be $R$-positive in $E$ if the set $L_{A}=\left\{\xi(A+\xi I)^{-1}: \xi \in S_{\varphi}\right\}$, $0 \leq \varphi<\pi$, is $R$-bounded.

Note that for Hilbert spaces $H_{1}, H_{2}$, all norm-bounded sets are $R$-bounded (see, e.g., [16]). Therefore, in Hilbert spaces all positive operators are $R$-positive. If $A$ is a generator of a contraction semigroup on $L_{q}, 1 \leq q \leq \infty$, or $A$ has the bounded imaginary powers with $\left\|A^{i t}\right\|_{B(E)} \leq C e^{v|t|}, v<\frac{\pi}{2}$ in $E \in \mathrm{UMD}$, then those operators are $R$-positive (e.g., see $[16$, Section 4.3]).

The operator $A(t), t \in \sigma \subset R$ is said to be $\varphi$-positive in $E$ uniformly with respect to $t \in \sigma$ if $D(A(t))$ is independent of $t, D(A(t))$ is dense in $E$ and $\left\|(A(t)+\lambda)^{-1}\right\| \leq \frac{M}{1+|\lambda|}$ for all $\lambda \in S(\varphi)$, $0 \leq \varphi<\pi$, where $M$ does not depend on $t$.

Let $E_{0}$ and $E$ be two Banach spaces and $E_{0}$ be continuously and densely embedded into $E$. Let $m$ be a positive integer. $W_{p, \gamma}^{m}\left(a, b ; E_{0}, E\right)$ denotes an $E_{0}$-valued function space defined by

$$
\begin{aligned}
& W_{p, \gamma}^{m}\left(a, b ; E_{0}, E\right)=\left\{u: u \in L_{p, \gamma}\left(a, b ; E_{0}\right), u^{(m)} \in L_{p, \gamma}(a, b ; E)\right\}, \\
& \|u\|_{W_{p, \gamma}^{m}}=\|u\|_{W_{p, \gamma}^{m}\left(a, b ; E_{0}, E\right)}=\|u\|_{L_{p, \gamma}\left(a, b ; E_{0}\right)}+\left\|u^{(m)}\right\|_{L_{p, \gamma}(a, b ; E)}<\infty .
\end{aligned}
$$

Let $t$ be a positive parameter. We define a parameterized norm in $W_{p, \gamma}^{m}\left(a, b ; E_{0}, E\right)$ as follows:

$$
\|u\|_{W_{p, \gamma, t}^{m}}=\|u\|_{W_{p, \gamma, t}^{m}\left(a, b ; E_{0}, E\right)}=\|u\|_{L_{p, \gamma}\left(a, b ; E_{0}\right)}+\left\|t u^{(m)}\right\|_{L_{p, \gamma}(a, b ; E)}<\infty .
$$

Let $\mathbf{p}=\left(p_{1}, p_{2}, \ldots, p_{n}\right), G=\prod_{k=1}^{n}\left(0, b_{k}\right), L_{\mathbf{p}}(G ; E)$ denote the space of all $\mathbf{p}$-summable $E$ valued functions with a mixed norm (see, e.g., [24, Section 8] for scalar case), i.e., the space of all measurable $E$-valued functions $f$ defined on $G$, for which

$$
\|f\|_{L_{\mathbf{p}}(G ; E)}=\left(\int_{0}^{b_{1}}\left(\int_{0}^{b_{2}} \cdots\left(\int_{0}^{b_{n}}\|f(x)\|^{p_{n}} d x_{n}\right)^{\frac{p_{n-1}}{p_{n}}} d x_{n-1}\right) \cdots d x_{1}\right)^{\frac{1}{p_{1}}}<\infty .
$$

Let $l_{k}$ be positive integers, $l=\left(l_{1}, l_{2}, \ldots, l_{n}\right), t_{k}$ be positive parameters and $t=\left(t_{1}, t_{2}, \ldots, t_{n}\right)$.

$$
D_{x_{k}}^{[i]} u=\left[x_{k}^{\alpha_{k}} \frac{\partial}{\partial x_{k}}\right]^{i} u(x), \quad \alpha_{k}>0, \alpha=\left(\alpha_{1}, \alpha_{2}, \ldots, \alpha_{n}\right), k=1,2, \ldots, n .
$$

Consider the following weighted spaces of functions:

$$
W_{\mathbf{p}, \alpha}^{[l]}(G ; E(A), E)=\left\{u: u \in L_{\mathbf{p}}(G ; E(A)), D_{k}^{\left[l_{k}\right]} u \in L_{\mathbf{p}}(G ; E)\right\},
$$


with the mixed norm

$$
\|u\|_{W_{\mathbf{p}, \alpha}^{[l]}(G ; E(A), E)}=\|u\|_{L_{\mathbf{p}}\left(G ; E_{0}\right)}+\sum_{k=1}^{n}\left\|D_{k}^{\left[l_{k}\right]} u\right\|_{L_{\mathbf{p}}(G ; E)}
$$

and with the parameterized norm

$$
\|u\|_{W_{\mathbf{p}, \alpha, t}^{[l]}(G ; E(A), E)}=\|u\|_{L_{\mathbf{p}}\left(G ; E_{0}\right)}+\sum_{k=1}^{n}\left\|t_{k} D_{k}^{\left[l_{k}\right]} u\right\|_{L_{\mathbf{p}}(G ; E)},
$$

respectively.

Consider the BVP for DOE

$$
\begin{aligned}
& (L+\lambda) u=(-1)^{m} t u^{[2 m]}(x)+(A+\lambda) u(x)=f(x), \quad x \in(0,1), \\
& L_{k} u=\sum_{i=0}^{m_{k}}\left[\alpha_{k i} u^{[i]}(1)+\sum_{i=1}^{N} \delta_{k i} u^{[i]}\left(x_{k i}\right)\right]=0,
\end{aligned}
$$

where $u^{[i]}=\left[x^{\gamma} \frac{d}{d x}\right]^{i} u(x), \alpha_{k i}, \delta_{k i}$ are complex numbers and $x_{k i} \in(0,1), m_{k} \in\{0,1, \ldots, 2 m-$ $1\} ; A$ is a possible unbounded operator in $E$.

In a similar way as in [17, Theorem 5.1], we obtain the following.

Theorem $\mathbf{A}_{\mathbf{1}}$ Let the following conditions be satisfied:

(1) $\alpha_{k i}, \delta_{k j}$ are complex numbers, $\alpha_{k m_{k}} \neq 0, t$ is a small positive parameter and $\sigma_{i}=\frac{1}{2 m p(\gamma-1)}+\frac{i}{2 m}$

(2) E is a UMD space, $\gamma>1+\frac{1+\sqrt{4 p+1}}{2 p}, 1<p<\infty$;

(3) $A$ is an R-positive operator in $E$.

Then problem (1) for $f \in L_{p}(0,1 ; E)$ and $|\arg \lambda| \leq \varphi$ with sufficiently large $|\lambda|$ has a unique solution $u \in W_{p, \gamma}^{[2 m]}(0,1 ; E(A), E)$. Moreover, the following uniform coercive estimate holds:

$$
\sum_{i=0}^{2 m}|\lambda|^{1-\frac{i}{2 m}} t^{\sigma_{i}}\left\|u^{[i]}\right\|_{L_{p}(0,1 ; E)}+\|A u\|_{L_{p}(0,1 ; E)} \leq C\|f\|_{L_{p}(0,1 ; E)} .
$$

By reasoning as in [17, Theorem 2.3], we obtain the following.

Theorem $\mathbf{A}_{2}$ Let the following conditions be satisfied:

(1) $\gamma(x)=x^{\frac{\gamma}{1-\gamma}}, \gamma>1+\frac{1+\sqrt{4 p+1}}{2 p}, p \in(1, \infty), 0<t<1$;

(2) $E$ is a UMD space and $A$ is an R-positive operator in $E$;

(3) there exists a bounded linear extension operator from $W_{p, \gamma}^{m}(1, \infty ; E(A), E)$ to $W_{p, \gamma}^{m}(R ; E(A), E)$.

Then the embedding $D^{j} W_{p, \gamma}^{m}(1, \infty ; E(A), E) \subset L_{p, \gamma}\left(1, \infty ; E\left(A^{1-\frac{j}{m}-\mu}\right)\right)$ is continuous and for $0 \leq \mu \leq 1-\frac{j}{m}$, the uniform estimate

$$
\left\|t^{\frac{j}{m}} u^{(j)}\right\|_{L_{p, \gamma}\left(1, \infty ; E\left(A^{1-\frac{j}{m}-\mu}\right)\right)} \leq h^{\mu}\|u\|_{W_{p, \gamma, t}^{m}(1, \infty ; E(A), E)}+h^{-(1-\mu)}\|u\|_{L_{p, \gamma}(1, \infty ; E)}
$$

holds for all $u \in W_{p, \gamma}^{m}(1, \infty ; E(A), E)$ and $0<h \leq h_{0}<\infty$. 
Let

$$
\begin{aligned}
& D^{[\alpha]}=D_{1}^{\left[\alpha_{1}\right]} D_{2}^{\left[\alpha_{2}\right]} \cdots D_{n}^{\left[\alpha_{n}\right]}, \quad t=\left(t_{1}, t_{2}, \ldots, t_{n}\right), \quad \psi(t)=\prod_{k=1}^{n} t_{k}^{\frac{\alpha_{k}}{2 m_{k}}}, \\
& 0<t_{k}<1, \quad G=\prod_{k=1}^{n}\left(0, b_{k}\right) .
\end{aligned}
$$

Theorem $\mathbf{A}_{3}$ Let the following conditions be satisfied:

(1) $\mathbf{p}=\left(p_{1}, p_{2}, \ldots, p_{n}\right), p_{k} \in(1, \infty), \gamma_{k}(x)=x_{k}^{\gamma_{k}}$;

(2) $E$ is a UMD space and $A$ is an R-positive operator in $E$;

(3) $\alpha=\left(\alpha_{1}, \alpha_{2}, \ldots, \alpha_{n}\right)$ and $l=\left(l_{1}, l_{2}, \ldots, l_{n}\right)$ are $n$-tuples of a nonnegative integer such that

$$
\varkappa=|\alpha: l|=\sum_{k=1}^{n} \frac{\alpha_{k}}{l_{k}} \leq 1, \quad 1<p_{k}<\infty, \quad \gamma_{k}>1+\frac{1+\sqrt{4 p+1}}{2 p}
$$

(4) there exists a bounded linear extension operator from $W_{\mathbf{p}, \gamma}^{[l]}(G ; E(A), E)$ to $W_{\mathbf{p}, \gamma}^{[l]}\left(R^{n} ; E(A), E\right)$.

Then the embedding $D^{\alpha} W_{\mathbf{p}, \gamma}^{[l]}(G ; E(A), E) \subset L_{\mathbf{p}}\left(G ; E\left(A^{1-\varkappa-\mu}\right)\right)$ is continuous. Moreover, there is a constant $h>0$ such that for $u \in W_{\mathbf{p}, \gamma}^{[l]}(\Omega ; E(A), E)$, the following uniform estimate holds:

$$
\psi(t)\left\|D^{[\alpha]} u\right\|_{L_{\mathbf{p}}\left(G ; E\left(A^{1-\varkappa-\mu}\right)\right)} \leq h^{\mu}\|u\|_{W_{\mathbf{p}, \gamma, t}^{[l]}(G ; E(A), E)}+h^{-(1-\mu)}\|u\|_{L_{\mathbf{p}}(G ; E)}
$$

\section{Singular degenerate DOEs with parameter}

Consider the BVP for the following differential-operator equation with parameter:

$$
\begin{aligned}
& L u=(-1)^{m} t a(x) u^{[2 m]}+A_{\lambda}(x) u+\sum_{k=0}^{2 m-1} t^{\frac{k}{2 m}} A_{k}(x) u^{[k]}=f(x), \\
& L_{k} u=\sum_{i=0}^{m_{k}}\left[\alpha_{k i} u^{[i]}(1)+\sum_{i=1}^{N} \delta_{k i} u^{[i]}\left(x_{k i}\right)\right]=0, \quad k=1,2, \ldots, m,
\end{aligned}
$$

on the domain $(0,1)$, where $t$ is a positive parameter and $\lambda$ is a complex parameter; $\alpha_{k i}$, $\delta_{k i}$ are complex numbers and $x_{k i} \in(0,1), m_{k} \in\left\{0,1, \ldots, 2 m_{k}-1\right\}, a(x)$ is a complex-valued function on [0,1]; $A(x)$ and $A_{k}(x)$ are linear operators in a Banach space $E$ and $A_{\lambda}(x)=$ $A(x)+\lambda$. Note that

$$
\int_{0}^{1} z^{-\gamma} d z=\infty
$$

A function $u \in W_{p, \gamma}^{[2 m]}(0,1 ; E(A), E)$ satisfying equation (2) a.e. on $(0,1)$ is said to be the solution of equation $(2)$ on $(0,1)$.

\section{Remark 1 Let}

$$
y=-\int_{x}^{1} z^{-\gamma} d z
$$


Under the substitution (3), spaces $L_{p}(0,1 ; E)$ and $W_{p, r}^{[2 m]}(0,1 ; E(A), E)$ are mapped isomorphically onto weighted spaces

$$
L_{p, \tilde{\gamma}}(-\infty, 0 ; E), \quad W_{p, \tilde{\gamma}}^{2}(-\infty, 0 ; E(A), E)
$$

respectively, where

$$
\tilde{\gamma}=\tilde{\gamma}(x(y))=[1-(\gamma-1) y]^{\frac{\gamma}{1-\gamma}} .
$$

Moreover, under the substitution (3), problem (2) is transformed into the following nondegenerate problem:

$$
\begin{aligned}
& L u=(-1)^{m} t a(y) u^{(2 m)}(y)+A_{\lambda}(y) u(y)+\sum_{k=0}^{2 m-1} t^{\frac{k}{2 m}} A_{k}(y) u^{(k)}(y)=f(y), \\
& L_{k} u=\sum_{i=0}^{m_{k}}\left[\alpha_{k i} u^{(i)}(0)+\sum_{i=1}^{N} \delta_{k i} u^{[i]}\left(y_{k i}\right)\right]=0, \quad k=1,2, \ldots, m
\end{aligned}
$$

in the weighted space $L_{p, \tilde{\gamma}}(-\infty, 0 ; E)$, where $\tilde{a}(y)=a(\tilde{\gamma}(x(y))), \tilde{A}(y)=A(\tilde{\gamma}(x(y))), \tilde{A}_{k}(y)=$ $A_{k}(\tilde{\gamma}(x(y))), \tilde{\gamma}(y)=\gamma(\tilde{\gamma}(x(y)))$ are again denoted by $a(y), A(y), A_{k}(y), \gamma$ after the substitution (3), respectively.

Let us consider boundary value problem (4)-(5).

Theorem 1 Let the following conditions be satisfied:

(1) $\alpha_{k m_{k}} \neq 0, a(y)$ is a positive uniformly bounded continuous function on $(-\infty, 0)$;

(2) $E$ is a UMD space, $\gamma>1+\frac{1+\sqrt{4 p+1}}{2 p}, 1<p<\infty$, and $\sigma_{i}=\frac{1}{2 m p(\gamma-1)}+\frac{i}{2 m}$;

(3) $A(y)$ is $R$-positive in $E$ uniformly with respect to $y \in[-\infty, 0]$ and $A(y) A^{-1}\left(y_{0}\right) \in$ $C((-\infty, 0) ; B(E)), y_{0} \in[-\infty, 0]$;

(4) for any $\varepsilon>0$, there is a positive $C(\varepsilon)$ such that

$$
\left\|A_{k}(y) u\right\| \leq \varepsilon\|u\|_{(E(A), E) \frac{k}{2 m}, \infty}+C(\varepsilon)\|u\| \quad \text { for } u \in(E(A), E)_{\frac{k}{2 m}, \infty}, k=0,1, \ldots, 2 m-1 .
$$

Then problem (4)-(5) has a unique solution $u \in W_{p, \gamma}^{2}(-\infty, 0 ; E(A), E)$ for $f \in L_{p, \tilde{\gamma}}(-\infty, 0$; $E)$ and $|\arg \lambda| \leq \varphi$ with sufficiently large $|\lambda|$. Moreover, the following uniform coercive estimate holds:

$$
\sum_{i=0}^{2 m}|\lambda|^{1-\frac{i}{2 m}} t^{\sigma_{i}}\left\|u^{(i)}\right\|_{p, \gamma}+\|A u\|_{p, \gamma} \leq C\|f\|_{p, \gamma} .
$$

Proof Let $G_{1}, G_{2}, \ldots$ be bounded intervals in $(-\infty, 0)$ and $\varphi_{1}, \varphi_{2}, \ldots$ correspond to a partition of unit that functions $\varphi_{j}$ are smooth on $(-\infty, 0), \sigma_{j}=\operatorname{supp} \varphi_{j} \subset G_{j}$ and $\sum_{j=1}^{\infty} \varphi_{j}(y)=1$. Then, for all $u \in W_{p, \gamma}^{2 m}(-\infty, 0 ; E(A), E)$, we have $u(y)=\sum_{j=1}^{\infty} u_{j}(y)$, where $u_{j}(y)=u(y) \varphi_{j}(y)$. For $u \in W_{p, \gamma}^{2}(-\infty, 0 ; E(A), E)$, from (4) we obtain

$$
(L+\lambda) u_{j}=(-1)^{m} t a(y) u_{j}^{(2 m)}(y)+A_{\lambda}(y) u_{j}(y)=f_{j}(y), \quad L_{k} u_{j}=0,
$$


where

$$
f_{j}=f \varphi_{j}-(-1)^{m} a \sum_{i=0}^{2 m-1} C_{2 m}^{i} u^{(i)} \varphi_{j}^{(2 m-i)}+\sum_{k=1}^{2 m-1} \sum_{i=0}^{k} C_{k}^{i} t^{\frac{k}{2 m}} u^{(i)} \varphi_{j}^{(k-i)} A_{k}
$$

Since $a$ is uniformly bounded on $(-\infty, 0)$ for all small $\rho>0$, there is a large $r_{0}>0$ such that $|a(y)-a(-\infty)| \leq \delta$ for all $|y| \geq r_{0}$. Let

$$
G_{0}=R^{n} \backslash \sigma_{r_{0}}(0), \quad \sigma_{r_{0}}(0)=\left\{y \in(-\infty, 0),|y| \leq r_{0}\right\} .
$$

Cover $\sigma_{r_{0}}(0)$ by finitely many intervals $G_{j}=\sigma_{r_{j}}\left(y_{0 j}\right)$ such that

$$
\left|a(y)-a\left(y_{0 j}\right)\right| \leq \delta \quad \text { for }\left|y-y_{0 j}\right| \leq r_{j}, j=1,2, \ldots
$$

Define coefficients of local operators, i.e.,

$$
\begin{aligned}
& a^{0}(y)=\left\{\begin{array}{ll}
a(y), & y \notin \sigma_{r_{0}}(0) \\
a\left(r_{0}^{2} \frac{y}{|y|^{2}}\right), & y \in \sigma_{r_{0}}(0)
\end{array}\right\}, \\
& a^{j}(y)=\left\{\begin{array}{ll}
a(y), & y \in \sigma_{r_{j}}\left(y_{0 j}\right) \\
a\left(y_{0 j}+r_{0}^{2} \frac{y-y_{0 j}}{\left|y-y_{0 j}\right|^{2}},\right. & y \notin \sigma_{r_{j}}\left(y_{0 j}\right)
\end{array}\right\}
\end{aligned}
$$

and

$$
\begin{aligned}
& A^{0}(y) A^{-1}\left(y_{0 j}\right)=\left\{\begin{array}{ll}
A(y) A^{-1}\left(y_{0 j}\right), & y \notin \sigma_{r_{0}}(0) \\
A\left(r_{0}^{2} \frac{y}{|y|^{2}}\right) A^{-1}\left(y_{0 j}\right), & y \in \sigma_{r_{0}}(0)
\end{array}\right\}, \\
& A^{j}(y) A^{-1}\left(y_{0 j}\right)=\left\{\begin{array}{ll}
A(y) A^{-1}\left(y_{0 j}\right), & y \in \sigma_{r_{j}}\left(y_{0 j}\right) \\
A\left(y_{0 j}+r_{0}^{2} \frac{y-y_{0 j}}{\left|y-y_{0 j}\right|^{2}}\right) A^{-1}\left(y_{0 j}\right), & y \notin \sigma_{r_{j}}\left(y_{0 j}\right)
\end{array}\right\}
\end{aligned}
$$

for each $j=1,2, \ldots$. Then, for all $y \in(-\infty, 0)$ and $j=0,1,2, \ldots$, we get

$$
\left|a^{j}(x)-a^{j}\left(y_{0 j}\right)\right| \leq \delta \quad \text { and } \quad\left\|A^{j}(y) A^{-1}\left(y_{0 j}\right)-A^{j}\left(y_{0 j}\right) A^{-1}\left(y_{0 j}\right)\right\|_{B(E)}<\delta \text {. }
$$

Freezing coefficients in (7) obtain that

$$
(-1)^{m} a\left(y_{0 j}\right) t u_{j}^{(2 m)}(y)+A_{\lambda}\left(y_{0 j}\right) u_{j}(y)=F_{j}(y), \quad L_{k} u_{j}=0,
$$

where

$$
F_{j}=f_{j}+\left[A\left(y_{0 j}\right)-A(y)\right] u_{j}+(-1)^{m} t\left[a(y)-a\left(y_{0 j}\right)\right] u_{j}^{(2 m)}, \quad j=1,2, \ldots
$$

Since functions $u_{j}(x)$ have compact supports in (9), if we extend $u_{j}(x)$ on the outsides of $\sigma_{j}=\operatorname{supp} \varphi_{j}$, we obtain BVPs with constant coefficients

$$
-t a\left(y_{0 j}\right) u_{j}^{(2 m)}+A_{\lambda}\left(y_{0 j}\right) u_{j}=F_{j}, \quad L_{k} u_{j}=0, \quad j=1,2, \ldots
$$


Let $\|\cdot\|_{G_{j}, p, \gamma}$ denote $E$-valued weighted $L_{p}$-norms with respect to domains $G_{j}$. Let $\varphi_{j}$ be such that $0 \in \sigma_{j}$. Then, by virtue of Theorem $\mathrm{A}_{1}$, we obtain that problem (11) has a unique solution $u_{j}$ and for $|\arg \lambda| \leq \varphi$ and sufficiently large $|\lambda|$, the following estimate holds:

$$
\sum_{i=0}^{2 m}|\lambda|^{1-\frac{i}{2 m}} t^{\sigma_{i}}\left\|u_{j}^{(i)}\right\|_{G_{j}, p, \gamma_{1}}+\left\|A u_{j}\right\|_{G_{j}, p, \gamma_{1}} \leq C\left\|F_{j}\right\|_{G_{j}, p, \gamma_{1}}
$$

Theorem $\mathrm{A}_{2}$ implies that for all $\varepsilon>0$, there is a continuous function $C(\varepsilon)$ such that

$$
\left\|F_{j}\right\|_{G_{j}, p, \gamma} \leq \varepsilon\left\|u_{j}\right\|_{W_{p, \gamma, t}^{2 m}\left(G_{j} ; E(A), E\right)}+C(\varepsilon)\left\|f_{j}\right\|_{G_{j}, p, \gamma}, \quad j=1,2, \ldots
$$

Consequently, by using Theorem $\mathrm{A}_{2}$, from (12)-(13) we get

$$
\begin{aligned}
& \sum_{i=0}^{2 m}|\lambda|^{1-\frac{i}{2 m}} t^{\sigma_{i}}\left\|u_{j}^{(i)}\right\|_{G_{j, p, \gamma}}+\left\|A u_{j}\right\|_{G_{j}, p, \gamma} \\
& \quad \leq C\|f\|_{G_{j, p, \gamma}}+\varepsilon\left\|u_{j}\right\|_{W_{p, \gamma}^{2 m}}+C(\varepsilon)\left\|u_{j}\right\|_{G_{j, p}, \gamma} .
\end{aligned}
$$

Choosing $\varepsilon<1$, from (14) we have

$$
\sum_{i=0}^{2 m}|\lambda|^{1-\frac{i}{2 m}} t^{\sigma_{i}}\left\|u_{j}^{(i)}\right\|_{G_{j}, p, \gamma}+\left\|A u_{j}\right\|_{G_{j}, p, \gamma} \leq C\left[\|f\|_{G_{j}, p, \gamma}+\left\|u_{j}\right\|_{G_{j}, p, \gamma}\right] .
$$

Then, by using the equality $u(y)=\sum_{j=1}^{\infty} u_{j}(y)$ and by virtue of (15) for $u \in W_{p, \gamma}^{2 m}(-\infty, 0 ; E(A)$, $E)$, we have

$$
\sum_{i=0}^{2 m}|\lambda|^{1-\frac{i}{2 m}} t^{\sigma_{i}}\left\|u^{(i)}\right\|_{p, \gamma}+\left\|A u_{j}\right\|_{p, \gamma} \leq C\left[\|(L+\lambda) u\|_{p, \gamma}+\|u\|_{p, \gamma}\right]
$$

Let $u \in W_{p, \gamma}^{2 m}(-\infty, 0 ; E(A), E)$ be a solution of problem (4)-(5). For $|\arg \lambda| \leq \varphi$, we have

$$
\|u\|_{p, \gamma}=\|(L+\lambda) u-L u\|_{p, \gamma} \leq \frac{1}{\lambda}\left[\|(L+\lambda) u\|_{p, \gamma}+\|u\|_{W_{p, \gamma}^{2 m}}\right] .
$$

By Theorem $A_{2}$, by virtue of (16) and (17) for sufficiently large $|\lambda|$, we have

$$
\sum_{i=0}^{2 m}|\lambda|^{1-\frac{i}{2 m}} t^{\sigma_{i}}\left\|u^{(i)}\right\|_{p, \gamma}+\|A u\|_{p, \gamma} \leq C\|(L+\lambda) u\|_{p, \gamma} .
$$

Consider the operator $O_{t \lambda}$ in $L_{p, \gamma}(-\infty, 0 ; E)$ generated by problem (4)-(5), i.e.,

$$
\begin{aligned}
& D\left(O_{\lambda}\right)=W_{p, \gamma}^{2 m}\left(-\infty, 0 ; E(A), E, L_{k}\right), \\
& O_{t \lambda} u=(-1)^{m} \operatorname{tau}^{(2 m)}+A_{\lambda} u+\sum_{k=0}^{2 m-1} t^{\frac{k}{2 m}} A_{k}(y) u^{(k)} .
\end{aligned}
$$

Estimate (18) implies that problem (4)-(5) has only a unique solution and the operator $O_{\lambda}$ has an invertible operator in its rank space. We need to show that this rank space 
coincides with the space $X=L_{p, \gamma}(-\infty, 0 ; E)$. We consider the smooth functions $g_{j}=g_{j}(y)$ with respect to the partition of the unit $\varphi_{j}=\varphi_{j}(y)$ on $(-\infty, 0)$ that equals one on $\operatorname{supp} \varphi_{j}$, where supp $g_{j} \subset G_{j}$ and $\left|g_{j}(y)\right|<1$. Let us construct for all $j$ the function $u_{j}$ that is defined on the regions $\Omega_{j}=(-\infty, 0) \cap G_{j}$ and satisfies problem (4)-(5). Problem (4)-(5) can be expressed in the form

$$
\begin{aligned}
& (-1)^{m} t a\left(y_{0 j}\right) u_{j}^{(2)}+A_{\lambda}\left(y_{0 j}\right) u_{j} \\
& \quad=g_{j}\left\{F_{j}+\left[A\left(y_{0 j}\right)-A(y)\right] u_{j}+(-1)^{m} t\left[a(y)-a\left(y_{0 j}\right)\right] u_{j}^{(2)}\right\}, \\
& L_{k} u_{j}=0, \quad j=1,2, \ldots
\end{aligned}
$$

Consider operators $O_{j t \lambda}$ in $L_{p, \gamma}\left(G_{j} ; E\right)$ generated by BVPs (19). By virtue of Theorem $\mathrm{A}_{1}$ for all $f \in L_{p, \gamma}\left(G_{j} ; E\right)$, for $|\arg \lambda| \leq \varphi$ and sufficiently large $|\lambda|$, we have

$$
\sum_{i=0}^{2 m}|\lambda|^{1-\frac{i}{2 m}} t^{\sigma_{i}}\left\|\frac{d^{i}}{d y^{i}} O_{j t \lambda}^{-1} f\right\|_{p, \gamma}+\left\|A O_{j t \lambda}^{-1} f\right\|_{p, \gamma} \leq C\|f\|_{p, \gamma} .
$$

Extending $u_{j}$ zero on the outside of $\operatorname{supp} \varphi_{j}$ in equalities (20) and passing substitutions $u_{j}=O_{j \lambda}^{-1} v_{j}$, we obtain operator equations with respect to $v_{j}$

$$
v_{j}=K_{j t \lambda} v_{j}+g_{j} f, \quad j=1,2, \ldots
$$

By virtue of Theorem $\mathrm{A}_{2}$, by estimate (20), in view of the smoothness of the coefficients of $K_{j \lambda t}$, for $|\arg \lambda| \leq \varphi$ and sufficiently large $|\lambda|$, we have $\left\|K_{j \lambda t}\right\|<\varepsilon$, where $\varepsilon$ is sufficiently small. Consequently, equations (21) have unique solutions

$$
v_{j}=\left[I-K_{j \lambda t}\right]^{-1} g_{j} f .
$$

Moreover,

$$
\left\|v_{j}\right\|_{p, \gamma}=\left\|\left[I-K_{j t \lambda}\right]^{-1} g_{j} f\right\|_{p, \gamma} \leq\|f\|_{p, \gamma} .
$$

Whence, $\left[I-K_{j \lambda t}\right]^{-1} g_{j}$ are bounded linear operators from $X$ to $L_{p, \gamma}\left(G_{j} ; E\right)$. Thus, we obtain that the functions

$$
u_{j}=U_{j t \lambda} f=O_{j t \lambda}^{-1}\left[I-K_{j t \lambda}\right]^{-1} g_{j} f
$$

are the solutions of equations (21). Consider the linear operator $\left(U_{t}+\lambda\right)$ in $X$ such that

$$
\left(U_{t}+\lambda\right) f=\sum_{j=1}^{\infty} \varphi_{j}(y) U_{j \lambda t} f
$$

It is clear from the constructions $U_{j t}$ and estimate (20) that operators $U_{j \lambda t}$ are bounded linear from $X$ to $W_{p, \gamma}^{2 m}(-\infty, 0 ; E(A), E)$ and

$$
\sum_{i=0}^{2 m}|\lambda|^{1-\frac{i}{2 m}} t^{\sigma_{i}}\left\|\frac{d^{i}}{d^{i} y} U_{j t \lambda}^{-1} f\right\|_{p, \gamma}+\left\|A U_{j t \lambda}^{-1} f\right\|_{p, \gamma} \leq C\|f\|_{p, \gamma} .
$$


Therefore, $\left(U_{t}+\lambda\right)$ is a bounded linear operator from $X$ to $X$. Let $\mathbf{L}_{t}$ denote the operator in $L_{p, \gamma}$ generated by BVP (4)-(5). Then the act of $\left(\mathbf{L}_{t}+\lambda\right)$ to $u=\sum_{j=1}^{\infty} \varphi_{j} U_{j t \lambda} f$ gives $\left(\mathbf{L}_{t}+\right.$ $\lambda) u=f+\sum_{j=1}^{\infty} \Phi_{j t \lambda} f$, where $\Phi_{j \lambda}$ is a linear combination of $U_{j \lambda}$ and $\frac{d}{d y} U_{j \lambda}$. By virtue of Theorem $\mathrm{A}_{2}$, estimate (22) and in view of the expression $\Phi_{j \lambda}$, we obtain that operators $\Phi_{j \lambda}$ are bounded linear from $X$ to $L_{p, \gamma}\left(G_{j} ; E\right)$ and $\left\|\Phi_{j \lambda t}\right\|<\varepsilon$. Therefore, there exists a bounded linear invertible operator $\left(I+\sum_{j=1}^{\infty} \Phi_{j t \lambda}\right)^{-1}$. Whence, we obtain that for all $f \in X$, BVP (4)(5) has a unique solution

$$
\begin{aligned}
u(y) & =\left(\mathbf{L}_{t}+\lambda\right)^{-1} f=\left(U_{t}+\lambda\right)\left(I+\sum_{j=1}^{\infty} \Phi_{j t \lambda}\right)^{-1} f \\
& =\sum_{j=1}^{\infty} \varphi_{j}(y) O_{j t \lambda}^{-1}\left[I-K_{j t \lambda}\right]^{-1}\left(I+\sum_{j=1}^{\infty} \Phi_{j t \lambda}\right)^{-1} f .
\end{aligned}
$$

Then, by using the above representation and in view of Theorem $A_{1}$, we obtain the assertion of Theorem 1 .

Result 1 Theorem 1 implies that the operator $\mathbf{L}_{t}$ has a resolvent $\left(\mathbf{L}_{t}+\lambda\right)^{-1}$ for $|\arg \lambda| \leq \varphi$ and the following estimate holds:

$$
\sum_{i=0}^{2 m}|\lambda|^{1-\frac{i}{2 m}} t^{\sigma_{i}}\left\|D^{i}\left(\mathbf{L}_{t}+\lambda\right)^{-1}\right\|_{B(X)}+\left\|A\left(\mathbf{L}_{t}+\lambda\right)^{-1}\right\|_{B(X)} \leq C .
$$

Let $\mathbf{G}_{t}$ denote the operator in $L_{p}(0,1 ; E)$ generated by BVP (2). By virtue of Theorem 1 and Remark 1 , we obtain the following result.

Result 2 Let all conditions of Theorem 1 be satisfied. Then

(a) problem (2) has a unique solution $u \in W_{p, \gamma}^{[2 m]}(0,1 ; E(A), E)$ for $f \in L_{p}(0,1 ; E)$ and sufficiently large $|\lambda|$. Moreover, the following uniform coercive estimate holds:

$$
\sum_{i=0}^{2 m}|\lambda|^{1-\frac{i}{2 m}} t^{\sigma_{i}}\left\|u^{[i]}\right\|_{p}+\|A u\|_{p} \leq C\|f\|_{p}
$$

(b) $\mathbf{G}_{t}$ has a resolvent operator $\left(\mathbf{G}_{t}+\lambda\right)^{-1}$ for $|\arg \lambda| \leq \varphi$ and

$$
\sum_{i=0}^{2 m}|\lambda|^{1-\frac{i}{2 m}} t^{\sigma_{i}}\left\|D^{[i]}\left(\mathbf{G}_{t}+\lambda\right)^{-1}\right\|_{B\left(L_{p}(0,1 ; E)\right)}+\left\|A\left(\mathbf{G}_{t}+\lambda\right)^{-1}\right\|_{B\left(L_{p}(0,1 ; E)\right)} \leq C .
$$

Theorem 2 Let all conditions of Theorem 1 hold. Then the operator $\mathbf{L}_{t}$ is uniformly $R$ positive in $L_{p}(0,1 ; E)$, also $\mathbf{L}_{t}$ is a generator of an analytic semigroup.

Proof By virtue of Theorem 1, we obtain that for $f \in L_{p}(0,1 ; E)$, BVP (4)-(5) has a unique solution expressed in the form

$$
\begin{aligned}
u(y) & =\left(\mathbf{L}_{t}+\lambda\right)^{-1} f=\left(U_{t}+\lambda\right)\left(I+\sum_{j=1}^{\infty} \Phi_{j \lambda t}\right)^{-1} f \\
& =\sum_{j=1}^{\infty} \varphi_{j}(y) O_{j t \lambda}^{-1}\left[I-K_{j t \lambda}\right]^{-1}\left(I+\sum_{j=1}^{\infty} \Phi_{j t \lambda}\right)^{-1} f
\end{aligned}
$$


where $O_{j \lambda t}=O_{j t}+\lambda$ are local operators generated by problems (7)-(8) and $K_{j t \lambda}, \Phi_{j t \lambda}$ are uniformly bounded operators in $L_{p}(0,1 ; E)$. In a similar way as in $[1,11,17]$, we obtain that operators $O_{j t}$ are $R$-positive. Then, by using the above representation and by virtue of Kahane's contraction principle, the product and additional properties of the collection of $R$-bounded operators (see, e.g., [16], Lemma 3.5, Proposition 3.4), we obtain the assertions.

\section{Singular degenerate anisotropic equation with parameters}

Consider the following degenerate BVP with parameters:

$$
\begin{aligned}
& \sum_{k=1}^{n}(-1)^{m_{k}} t_{k} a_{k}(x) D_{x_{k}}^{\left[2 m_{k}\right]} u+\sum_{|\beta: 2 m|<1} \prod_{k=1}^{n} t_{k}^{\frac{\alpha_{k}}{2 m_{k}}} A_{\beta}(x) D^{[\beta]} u+A(x)+\lambda u=f(x), \\
& L_{k j} u=\sum_{i=0}^{m_{k j}} \alpha_{k j i} u_{x_{k}}^{[i]}\left(G_{k b}\right)=0, \quad j=1,2, \ldots, m_{k}, k=1,2, \ldots, n,
\end{aligned}
$$

where $A(x)$ and $A_{\alpha}(x)$ are linear operators in a Banach space $E$,

$$
\begin{aligned}
& G=\left\{x=\left(x_{1}, x_{2}, \ldots, x_{n}\right), 0<x_{k}<b_{k}, k=1,2, \ldots, n\right\}, \\
& \beta=\left(\beta_{1}, \beta_{2}, \ldots, \beta_{n}\right), \quad m=\left(m_{1}, m_{2}, \ldots, m_{n}\right), \quad|\beta: 2 m|=\sum_{k=1}^{n} \frac{\beta_{k}}{2 m_{k}}, \\
& \gamma_{k}>1, \quad G_{k b}=\left(x_{1}, x_{2}, \ldots, x_{k-1}, b_{k}, x_{k+1}, \ldots, x_{n}\right), \\
& G_{k b}=\left(x_{1}, x_{2}, \ldots, x_{k-1}, b_{k}, x_{k+1}, \ldots, x_{n}\right), \quad 0 \leq m_{k j} \leq 2 m_{k}-1,
\end{aligned}
$$

$a_{k}$ are complex-valued functions on $G, \alpha_{k j i}$ are complex numbers, $t_{k}$ are positive and $\lambda$ is a complex parameter.

Note that BVP (24) is degenerated with different speeds on different directions in general.

The main result of this section is the following.

Theorem 3 Assume the following conditions hold:

(1) $E$ is a UMD space, $A(x)$ is R-positive in $E$ uniformly with respect to $x \in G$ and $A(x) A^{-1}\left(x_{0}\right) \in C(\bar{G} ; B(E)), x_{0} \in[-\infty, 0]$;

(2) for any $\varepsilon>0$, there is a positive $C(\varepsilon)$ such that

$$
\left\|A_{\beta}(x) u\right\| \leq \varepsilon\|u\|_{(E(A), E)_{1-|\beta: 2 m|, \infty}}+C(\varepsilon)\|u\| \quad \text { for } u \in(E(A), E)_{1-|\alpha: 2 m|, \infty}
$$

(3) $\gamma_{k}>1+\frac{1+\sqrt{4 p+1}}{2 p}, \sigma_{k i}=\frac{1}{2 m_{k} p\left(\alpha_{k}-1\right)}+\frac{i}{2 m_{k}}, i=0,1, \ldots, 2 m_{k}, p_{k} \in(1, \infty)$;

(4) $a_{k}$ are continuous positive functions on $\bar{G}$.

Then, for $f \in L_{\mathbf{p}}(G ; E),|\arg \lambda| \leq \varphi$ and sufficiently large $|\lambda|$, problem (24) has a unique solution $u$ that belongs to $W_{\mathbf{p}, \alpha}^{[2 m]}(G ; E(A), E)$ and the following coercive uniform estimate holds:

$$
\sum_{k=1}^{n} \sum_{i=0}^{2 m}|\lambda|^{1-\frac{i}{2 m}} t_{k}^{\sigma_{k i}}\left\|D_{x_{k}}^{[i]} u\right\|_{L_{\mathbf{p}}(G ; E)}+\|A u\|_{L_{\mathbf{p}}(G ; E)} \leq C\|f\|_{L_{\mathbf{p}}(G ; E)}
$$


Proof Consider the BVP

$$
\begin{aligned}
& (-1)^{m_{1}} a_{1}\left(x_{1}\right) t_{1} D_{x_{1}}^{\left[2 m_{1}\right]} u\left(x_{1}\right)+A(x) u\left(x_{1}\right)+\lambda u\left(x_{1}\right)=f\left(x_{1}\right), \\
& x_{1} \in\left(0, b_{1}\right), \quad L_{1 k} u=0, \quad k=1,2, \ldots, m_{1},
\end{aligned}
$$

where $L_{1 k}$ are boundary conditions of type (24) on $\left(0, b_{1}\right)$. By virtue of Result 2, problem (26) has a unique solution $u \in W_{p_{1}, \alpha_{1}}^{[2]}\left(0, b_{1} ; E(A), E\right)$ for all $f \in L_{p_{1}}\left(0, b_{1} ; E\right),|\arg \lambda| \leq \varphi$ and sufficiently large $|\lambda|$. Moreover, the following coercive uniform estimate holds:

$$
\sum_{i=0}^{2 m_{1}}|\lambda|^{1-\frac{i}{2 m_{1}}} t_{1}^{\sigma_{1 i}}\left\|u^{[i]}\right\|_{L_{p_{1}}\left(0, b_{1} ; E\right)}+\|A u\|_{L_{p_{1}}\left(0, b_{1} ; E\right)} \leq C\|f\|_{L_{p_{1}}\left(0, b_{1} ; E\right)} .
$$

Let us now consider in $L_{p_{1}, p_{2}}\left(G_{2} ; E\right)$ the BVP on the domain $G_{2}$

$$
\begin{aligned}
& \sum_{k=1}^{2}(-1)^{m_{k}} t_{k} a_{k}(x) D_{x_{k}}^{\left[2 m_{k}\right]} u+\lambda u=f\left(x_{1}, x_{2}\right), \\
& L_{k j} u=0, \quad j=1,2, \ldots, m_{k}, k=1,2,
\end{aligned}
$$

where $G_{2}=\left(0, b_{1}\right) \times\left(0, b_{2}\right)$. Since $L_{p_{2}}\left(0, b_{2} ; L_{p_{1}}\left(0, b_{1} ; E\right)\right)=L_{p_{1}, p_{2}}\left(G_{2} ; E\right)$, then problem $(27)$ can be expressed in the following way:

$$
(-1)^{m_{2}} t_{2} a_{2} D_{x_{2}}^{\left[2 m_{2}\right]} u\left(x_{2}\right)+(B+\lambda) u\left(x_{2}\right)=f\left(x_{2}\right), \quad L_{2 k} u=0,
$$

where $B$ is the differential operator in $L_{p_{1}}\left(0, b_{1} ; E\right)$ generated by problem (26), i.e.,

$$
D(B)=W_{p_{1} \alpha_{1}}^{\left[2 m_{1}\right]}\left(0, b_{1} ; L_{1 k}\right), \quad B u=a_{1}\left(x_{1}\right) t_{2} D^{2} u\left(x_{1}\right)+d u\left(x_{1}\right)
$$

By virtue of [22], $L_{p_{1}}\left(0, b_{1} ; E\right) \in \mathrm{UMD}$ for $p_{1} \in(1, \infty)$ provided $E \in \mathrm{UMD}$. Moreover, by virtue of Theorem 2, the operator $B$ is $R$-positive in $L_{p_{1}}\left(0, b_{1} ; E\right)$. Hence, by Result 2 , we get that problem (27) has a unique solution

$$
u \in W_{p_{1}, p_{2}, \tilde{\alpha}}^{[2]}\left(G_{2} ; E(A), E\right), \quad \tilde{\alpha}=\left(\alpha_{1}, \alpha_{2}\right)
$$

for $f \in L_{p_{1}, p_{2}}\left(G_{2} ; E\right),|\arg \lambda| \leq \varphi$ and sufficiently large $|\lambda|$, and coercive uniform estimate (25) holds. By continuing this process for $k=n$, we obtain that the following problem:

$$
\begin{aligned}
& \sum_{k=1}^{n}(-1)^{m_{k}} t_{k} a_{k}(x) D_{x_{k}}^{\left[2 m_{k}\right]} u+A(x) u+\lambda u=f(x), \\
& L_{k j} u=0, \quad j=1,2, \ldots, m_{k}, k=1,2, \ldots, n,
\end{aligned}
$$

for $f \in L_{\mathbf{p}}(G ; E),|\arg \lambda| \leq \varphi$ and sufficiently large $|\lambda|$, has a unique solution $u \in W_{\mathbf{p}, \alpha}^{[2 m]}(G$; $E(A), E)$ and the following coercive uniform estimate holds:

$$
\sum_{k=1}^{n} \sum_{i=0}^{2 m}|\lambda|^{1-\frac{i}{2 m}} t_{k}^{\sigma_{k i}}\left\|D_{x_{k}}^{[i]} u\right\|_{L_{\mathbf{p}}(G ; E)}+\|A u\|_{L_{\mathbf{p}}(G ; E)} \leq C\|f\|_{L_{\mathbf{p}}(G ; E)} .
$$


Moreover, by virtue of embedding Theorem $\mathrm{A}_{3}$, we have the Ehrling-Nirenberg-Gagliardo type estimate

$$
\left\|L_{1} u\right\|_{L_{\mathbf{p}}(G ; E)} \leq h^{\mu}\|u\|_{W_{\mathbf{p}, \alpha}^{[2]}(G ; E(A), E)}+h^{-(1-\mu)}\|u\|_{L_{\mathbf{p}}(G ; E)}, \quad h \in(0,1) .
$$

Let $Q_{t}$ denote the operator generated by problem (28) and

$$
L_{1} u=\sum_{|\beta: 2 m|<1} \prod_{k=1}^{n} t_{k}^{\frac{\beta_{k}}{2 m_{k}}} A_{\beta}(x) D^{[\beta]} u \text {. }
$$

By using estimate (29), we obtain that there is a $\delta \in(0,1)$ such that

$$
\left\|L_{1}\left(Q_{t}+\lambda\right)^{-1}\right\|_{L\left(L_{\mathbf{p}}(G ; E)\right)}<\delta
$$

Then, by using perturbation elements, we obtain the assertion.

From Theorem 2 and Theorem 3, we obtain the following result.

Result 3 Let all conditions of Theorem 3 hold for $\varphi>\frac{\pi}{2}$ and $A_{\alpha}=0$. Then the operator $Q_{t}$ is uniformly $R$-positive in $L_{p}(G ; E)$, it also is a generator of an analytic semigroup.

\section{Singular degenerate parabolic DOE}

Consider the following mixed problem for a parabolic DOE with parameter:

$$
\begin{aligned}
& \frac{\partial u}{\partial y}+\sum_{k=1}^{n}(-1)^{m_{k}} t_{k} a_{k}(x) D_{x_{k}}^{\left[2 m_{k}\right]} u+(A(x)+d) u=f(y, x), \quad y \in R_{+}, x \in G \\
& L_{k j} u=\sum_{i=0}^{m_{k j}} \alpha_{k j i} u_{x_{k}}^{[i]}\left(G_{k b}\right)=0, \quad j=1,2, \ldots, m_{k}, \\
& u(0, x)=0
\end{aligned}
$$

where $t_{k}, a_{k}(x), G, G_{k b}, m_{k j}$ are defined as in Section $3, d>0$.

For $\tilde{\mathbf{p}}=\left(p_{1}, p_{2}, \ldots, p_{n}, p_{0}\right), \Delta_{+}=R_{+} \times G, L_{\tilde{\mathbf{p}}}\left(\Delta_{+} ; E\right)$ will denote the space of all $E$-valued $\tilde{\mathbf{p}}$-summable functions with a mixed norm. Analogously,

$$
W_{\tilde{\mathbf{p}}, \gamma}^{[1,2 m]}\left(\Delta_{+}, E(A), E\right)
$$

denotes the Sobolev space with a corresponding mixed norm (see [24] for a scalar case).

Let $Q_{t}$ denote a differential operator generated by (28) for $\lambda=0$.

Theorem 4 Let all conditions of Theorem 3 hold for $A_{\beta}=0$ and $\varphi>\frac{\pi}{2}$. Then, for $f \in$ $L_{\tilde{\mathbf{p}}}\left(\Delta_{+} ; E\right)$ and sufficiently large $d>0$, problem (30) has a unique solution belonging to $W_{\tilde{\mathbf{p}}, \alpha}^{1,[2 m]}\left(\Delta_{+} ; E(A), E\right)$ and the following coercive estimate holds:

$$
\left\|\frac{\partial u}{\partial y}\right\|_{L_{\tilde{\mathbf{p}}}\left(G_{+} ; E\right)}+\sum_{k=1}^{n} t_{k}\left\|D_{x_{k}}^{\left[2 m_{k}\right]} u\right\|_{L_{\tilde{\mathbf{p}}\left(G_{+} ; E\right)}}+\|A u\|_{L_{\tilde{\mathbf{p}}}\left(G_{+} ; E\right)} \leq C\|f\|_{L_{\tilde{\mathbf{p}}}\left(G_{+} ; E\right)} .
$$


Proof Problem (30) can be expressed as the following Cauchy problem:

$$
\frac{d u}{d y}+\left(Q_{t}+d\right) u(y)=f(y), \quad u(0)=0 .
$$

Result 3 implies that the operator $Q_{t}$ is $R$-positive in $F=L_{\mathbf{p}}(G ; E)$. By [23, Section 1.14], $Q_{t}$ is a generator of an analytic semigroup in $F$. Then, by virtue of [20, Theorem 4.2], we obtain that for $f \in L_{p_{0}}\left(R_{+} ; F\right)$ problem (31) has a unique solution belonging to $W_{p_{0}}^{1}\left(R_{+}\right.$; $\left.D\left(Q_{t}\right), F\right)$ and the following estimate holds:

$$
\left\|\frac{d u}{d y}\right\|_{L_{p_{0}}\left(R_{+} ; F\right)}+\left\|Q_{t} u\right\|_{L_{p_{0}}\left(R_{+} ; F\right)} \leq C\|f\|_{L_{p_{0}}\left(R_{+} ; F\right)}
$$

Since $L_{p_{0}}\left(R_{+} ; F\right)=L_{\tilde{\mathbf{p}}}\left(\Delta_{+} ; E\right)$, by Theorem 3 we have

$$
\left\|\left(Q_{t}+d\right) u\right\|_{L_{p_{0}}\left(R_{+} ; F\right)}=D\left(Q_{t}\right) .
$$

The above estimate proves the hypothesis to be true.

\section{Cauchy problem for infinite systems of degenerate parabolic equations with small parameters}

Consider the infinity systems of BVP for the degenerate anisotropic parabolic equation:

$$
\begin{aligned}
& \frac{\partial u_{i}}{\partial y}+\sum_{k=1}^{n}(-1)^{m_{k}} t_{k} a_{k}(x) D_{x_{k}}^{\left[2 m_{k}\right]} u_{i}+\sum_{j=1}^{N} a_{i j}(x) u_{j}+d u=f_{i}(x, y), \quad i=1,2, \ldots, N, \\
& L_{k j} u=\sum_{i=0}^{m_{k j}} \beta_{k j v} D_{x_{k}}^{(v)} u_{i}\left(G_{k b}, y\right)=0, \quad j=1,2, \ldots, m_{k} \\
& u(0, x)=0, \quad x \in G
\end{aligned}
$$

where $N$ is finite or infinite natural number, $t_{k}, a_{k}(x), G, G_{k b}, G_{+}, m_{k j}, d$ are defined as in Sections 3 and $4, a_{i j}(x)$ are real functions and

$$
\begin{aligned}
& A(x)=\left\{a_{i j}(x)\right\}, \quad u=\left\{u_{j}\right\}, \quad A u=\left\{\sum_{j=1}^{N} a_{i j}(x) u_{j}\right\}, \quad i, j=1,2, \ldots, N, \\
& l_{q}(A)=\left\{u \in l_{q},\|u\|_{l_{q}(A)}=\|A u\|_{l_{q}}=\sup _{i}\left(\sum_{j=1}^{N}\left|a_{i j}(x) u_{j}\right|^{q}\right)^{\frac{1}{q}}<\infty\right\} .
\end{aligned}
$$

From Theorem 4 we obtain the following.

Theorem 5 Let $\gamma_{k}>1+\frac{1+\sqrt{4 p+1}}{2 p}, p_{k}, p_{0} \in(1, \infty), a_{k}, a_{i j} \in C(\bar{G}), a_{k}(x)>0, a_{i j} \neq 0$ and $a_{i j}=a_{j i}$. Then for $f(x, y)=\left\{f_{i}(x, y)\right\}_{1}^{N} \in L_{\tilde{\mathbf{p}}}\left(G_{+} ; l_{q}\right)$ and sufficiently large $d>0$, problem (32) has a unique solution $u=\left\{u_{i}(x, y)\right\}_{1}^{N}$ that belongs to the space $W_{\tilde{\mathbf{p}}, \alpha}^{1,[2 m]}\left(G_{+}, l_{q}(A), l_{q}\right)$ and the following coercive uniform estimate holds:

$$
\left\|\frac{\partial u}{\partial y}\right\|_{L_{\tilde{\mathbf{p}}}\left(G_{+} ; l_{q}\right)}+\sum_{k=1}^{n} t_{k}\left\|D_{x_{k}}^{\left[2 m_{k}\right]} u\right\|_{L_{\tilde{\mathbf{p}}}\left(G_{+} ; l_{q}\right)}+\|A u\|_{L_{\tilde{\mathbf{p}}}\left(G_{+} ; l_{q}\right)} \leq C\|f\|_{L_{\tilde{\mathbf{p}}}\left(G_{+} ; l_{q}\right)} .
$$


Proof Let $E=l_{q}$ and $A$ be infinite matrices such that

$$
A=\left[a_{i j}\right], \quad i, j=1,2, \ldots, N \text {. }
$$

It is clear that the operator $A$ is $R$-positive in $l_{q}$. Problem (32) can be rewritten as problem (30). Then, from Theorem 4, we obtain the assertion.

\section{Competing interests}

The authors declare that they have no competing interests.

\section{Authors' contributions}

The results to proving the uniform coercivity properties of ordinary and partial differential equations with small parameters in abstract $L_{p}$ spaces, the showing that corresponding differential operators are positive and also are generators of analytic semigroups and well-posedeness of Cauchy problem for abstract parabolic equation and systems of parabolic equations are studied in mixed $L_{\mathbf{p}}$ spaces due to VS.

The applications of these abstract problems to concrete mathematics and engineering problem belongs to AS. Both authors read and approved the final manuscript.

\section{Author details}

'Department of Environmental Sciences, Okan University, Akfirat, Tuzla, Istanbul 34959, Turkey. ${ }^{2}$ Department of Mechanical Engineering, Okan University, Akfirat, Tuzla, Istanbul 34959, Turkey.

\section{Acknowledgements}

Dedicated to Professor Hari M Srivastava.

Received: 22 November 2012 Accepted: 5 February 2013 Published: 15 February 2013

\section{References}

1. Agarwal, RP, Bohner, R, Shakhmurov, VB: Maximal regular boundary value problems in Banach-valued weighted spaces. Bound. Value Probl. 1, 9-42 (2005)

2. Ashyralyev, A: On well-posedeness of the nonlocal boundary value problem for elliptic equations. Numer. Funct. Anal. Optim. 24(1 \& 2), 1-15 (2003)

3. Dore, C, Yakubov, S: Semigroup estimates and non coercive boundary value problems. Semigroup Forum 60, 93-121 (2000)

4. Favini, A, Shakhmurov, V, Yakubov, Y: Regular boundary value problems for complete second order elliptic differential-operator equations in UMD Banach spaces. Semigroup Forum 79(1), 22-54 (2009)

5. Goldstain, JA: Semigroups of Linear Operators and Applications. Oxford University Press, Oxford (1985)

6. Krein, SG: Linear Differential Equations in Banach Space. Am. Math. Soc., Providence (1971)

7. Lunardi, A: Analytic Semigroups and Optimal Regularity in Parabolic Problems. Birkhäuser, Basel (2003)

8. Lions, J-L, Magenes, E: Nonhomogenous Boundary Value Problems. Mir, Moscow (1971)

9. Sobolevskii, PE: Coerciveness inequalities for abstract parabolic equations. Dokl. Akad. Nauk SSSR 57(1), 27-40 (1964)

10. Prüss, J: Maximal regularity for evolution equations in $L_{p}$-spaces. In: Lectures Given at the Summer School, Positivity and Semigroups, Monopoli (2002)

11. Shakhmurov, VB: Linear and nonlinear abstract equations with parameters. Nonlinear Anal., Theory Methods Appl. 73, 2383-2397 (2010)

12. Shakhmurov, VB: Imbedding theorems and their applications to degenerate equations. Differ. Equ. 24(4), 475-482 (1988)

13. Shakhmurov, VB: Coercive boundary value problems for regular degenerate differential-operator equations. J. Math. Anal. Appl. 292(2), 605-620 (2004)

14. Yakubov, S, Yakubov, Y: Differential-Operator Equations. Ordinary and Partial Differential Equations. Chapman and Hall/CRC, Boca Raton (2000)

15. Amann, H: Linear and Quasi-Linear Equations, vol. 1. Birkhäuser, Basel (1995)

16. Denk, R, Hieber, M, Prüss, J: R-boundedness, Fourier multipliers and problems of elliptic and parabolic type. Mem. Amer. Math. Soc. 166(788) (2003)

17. Shakhmurov, VB: Degenerate differential operators with parameters. Abstr. Appl. Anal. 2006, 1-27 (2007)

18. Shakhmurov, VB: Separable anisotropic differential operators and applications. J. Math. Anal. Appl. 327(2), 1182-1201 (2006)

19. Shakhmurov, VB: Nonlinear abstract boundary value problems in vector-valued function spaces and applications. Nonlinear Anal., Theory Methods Appl. 67(3), 745-762 (2006)

20. Weis, L: Operator-valued Fourier multiplier theorems and maximal $L_{p}$ regularity. Math. Ann. 319, 735-758 (2001)

21. Agarwal, R, O'Regan, D, Shakhmurov, VB: Separable anisotropic differential operators in weighted abstract spaces and applications. J. Math. Anal. Appl. 338, 970-983 (2008)

22. Burkholder, DL: A geometrical condition that implies the existence certain singular integral of Banach space-valued functions. In: Proc. Conf. Harmonic Analysis in Honor of Antonu Zigmund, Chicago, 1981, pp. 270-286. Wadsworth, Belmont (1983)

23. Triebel, H: Interpolation Theory, Function Spaces, Differential Operators. North-Holland, Amsterdam (1978)

24. Besov, OV, Ilin, VP, Nikolskii, SM: Integral Representations of Functions and Embedding Theorems. Nauka, Moscow (1975) 
doi:10.1186/1687-2770-2013-30

Cite this article as: Sahmurova and Shakhmurov: Singular degenerate problems occurring in biosorption process. Boundary Value Problems 2013 2013:30.

Submit your manuscript to a SpringerOpen ${ }^{\circ}$ journal and benefit from:

- Convenient online submission

- Rigorous peer review

- Immediate publication on acceptance

Open access: articles freely available online

- High visibility within the field

- Retaining the copyright to your article

Submit your next manuscript at $\gg$ springeropen.com 